\section{P15 (continued)}

\section{Study Design, Setting, Participants, Intervention:} A descriptive cross sectional survey was conducted among 1,610 undergraduates using multistage sampling in five universities, South East, Nigeria.

Outcome Measures and Analysis: A self-administered lifestyle questionnaire was used to collect information on demographics and MCVRFs such as physical inactivity, alcohol intake, smoking and consumption of unhealthy foods ( $\geq 3$ times a week). Other CVD risk factors such as waist circumference (WC), waist hip ratio (WHR) and blood pressure were assessed using standardized instruments. MCVRFs were defined as obesity (BMI $\geq 30 \mathrm{~kg} / \mathrm{m} 2$ ), overweight (BMI 25-29.9 kg/m2), abdominal obesity using World Health Organisation (WHO) cut offs for WC $\geq 94 \mathrm{~cm}$ in men and $\geq 80 \mathrm{~cm}$ in women and WHR $>0.90$ in men and 0.85 in women and hypertension (systolic/diastolic blood pressure $\geq 140 / 90 \mathrm{mmHg}$ ). Chi square was used for comparison of proportions between males and females. Results: The age range was 18-22 years (51.9\%). Prevalence of MCVRFs were obesity $20.6 \%$, overweight $13.4 \%$, WC 15.8\%, WHR 8.9\%, hypertension (SPB = 7.3\%; $\mathrm{DPB}=10.9 \%)$, consumption of unhealthy foods $(\geq 3$ times per week) (58.1\%), low fruit and vegetable intake (73.8\%), physical inactivity $(40,2 \%)$, alcohol intake (48.9\%) and smoking (47.6\%). Diastolic blood pressure (55.7\% vs. $44.3 \%$; $P=.007)$ and smoking (57.7\% vs. $2.3 \%)$ were significantly higher in males. Females had significantly higher BMI (70.2\% vs. 29.8\%; $P=.001)$, WC (94.\% vs. $5.6 \%$; $P=.001)$, WHR (92.9\% vs. $7.1 \% ; P=.001)$ and consumption of unhealthy foods $(57.5 \%$ vs. $42.5 \% ; P=0.001)$.

Conclusions and Implications: The high rate of MCVRFs among the undergraduates underscores the need for CVD prevention programs that focus on improving healthy lifestyle to reduce burden of the disease in later life.

Funding: None.

\section{P16 Perception of Weight Status is Associated with Consumption of Fruits and Vegetables by Adolescents}

Teresia Mbogori,PhD, tnmbogori@bsu.edu, Ball State University, $2000 \mathrm{~W}$ University Avenue, Muncie, IN 47306; Tya Arthur, PhD, MPH; Kylie Mennel, BS

Background (Background, Rationale, Prior Research, and/or Theory): Childhood obesity is a major concern in the United States with $20.6 \%$ of all children aged 12-19 years considered obese in 2017. Adequate consumption of fruits and vegetables is considered protective against childhood obesity.

Objective: To determine whether adolescents' perception of their weight status is associated with the frequency of consumption of fruits and vegetables.

Study Design, Setting, Participants, Intervention: A cross-sectional study was conducted with 1,072 adolescents (12-17 years) participating in the Family Life, Activity, Sun, Health, and Eating (FLASHE) Study, an internetbased survey study sponsored by the National Cancer Institute.
Outcome Measures and Analysis: Adolescents reported their perception of their weight status on a 5-point scale from "very overweight" to "very underweight". In addition, the adolescents reported the frequency with which they consumed common fruits and vegetables in the seven days preceding the study on a 6-point scale ranging from "none in the seven days" to "more than 3 times a day." Analysis of Variance (ANOVA) statistics were used to identify significant differences in the frequency of consumption of fruits and vegetables between the different weight categories. Significance was $P<.05$.

Results: Sixty-two point one percent of the adolescents reported perceiving their weight as "just right", 9.6\%, 22.4\% and, $4.7 \%$ perceived their weight to be "underweight", "a little overweight", and "very overweight" respectively. Those who perceived their weight status as "just right" also reported consuming significantly more fruits and vegetables than those who perceived their weight as "underweight" or "overweight" (mean $2.45 \pm 0.03$ vs. $2.28 \pm 0.06$ in underweight and $2.26 \pm 0.04$ in overweight). The frequency of consumption for both underweight and overweight groups was not significantly different.

Conclusions and Implications: Although adolescents who perceived their weight to be "just right" consumed fruits and vegetables more frequently than their underweight and overweight counterparts, the average consumption was very low in all weight status groups. Interventions should focus on identifying and managing barriers to consumption of fruits and vegetables by adolescents regardless of the weight status.

Funding: None.

\section{P17 Promoting Farmers' Markets with Limited-Resource Audiences: The Impact of a Social Marketing Campaign}

ChristopherSneed,PhD, csneed@utk.edu, University of Tennessee Extension, 119 Morgan Hall, 2621 Morgan Circle, Knoxville, TN 37919; Karen Franck, PhD; Janie Burney, PhD, RD

Background (Background, Rationale, Prior Research, and/or Theory): Farmers' markets can increase access to locally-grown produce for limited-resource consumers. However, studies have identified several barriers for farmers' market patronage by these consumers. Social marketing campaigns have the potential to address these barriers. These campaigns are most effective when messages and materials are tailored to specific audiences.

Objective: To examine the impact of a farmers' market social marketing campaign on limited-resource consumers' intent to purchase and consume locally-grown produce and to examine potential differences in campaign impacts based on consumers' communities of residence (rural, suburban, urban).

Study Design, Setting, Participants, Intervention: In 2016, a farmers' market social marketing campaign was implemented in 33 counties at 39 farmers' 